\title{
Can eating harder textured foods slowly influence appetite?
}

\section{Abstract \\ Introduction}

Chewing a greater number of chews per bite has been shown to increase fullness, lower hunger levels and lead to a lower energy intake, when compared to chewing each bite fewer times. Increased levels of fullness and decreased levels of hunger have also been observed after consuming harder textured foods which require more chewing activity and have a longer oro-sensory exposure time. The aim of this study was to investigate whether consumption of foods differing in texture, classified as 'fast' or 'slow' foods, combined with differences in oral processing behaviours (chewing normally or more slowly), has an impact on self-reported hunger, fullness and satiety outcomes.

\section{Materials and Methods}

This is an interim analysis of a randomised crossover experiment designed to test the impact of both oral processing behaviours and food texture on self-reported hunger, fullness and satiety. Participants consumed two breakfasts of different texture (a 'fast' yoghurt and fruit compote option, and a 'slow' granola option) twice; once at their normal rate of eating and once after being instructed to eat slowly. Measures of self-reported appetite (hunger, fullness, satiety, desire to eat and prospective food consumption) were assessed using a visual analogue scale at three time points; baseline (minimum 20 minutes before consumption), immediately pre-consumption and post-consumption. Statistical analysis was conducted in SPSS; paired sample t tests and one-way analysis of variance was conducted to measure whether change in appetite ratings between the time points differed between the four breakfast options.

\section{Results}

Data were collected from 16 healthy volunteers (study aiming to recruit $n=21$; mean age $32.1 \mathrm{y}$, female $n=13,81.3 \%$ ). For all four breakfast options, levels of hunger, desire to eat and prospective food consumption significantly decreased, and levels of fullness and satiety significantly increased between both baseline and post-consumption, $(p<0.05)$ and between pre-consumption and postconsumption $(\mathrm{p}<0.05)$. However, amongst the four breakfast options, no significant differences were observed in change in these appetite measures between baseline and post-consumption and pre- and post-consumption $(\mathrm{p}>0.05)$.

\section{Discussion}

This interim analysis demonstrates no differences in mean change in appetite measures for breakfast options of different texture chewed normally or more slowly. Analysis of the full study will determine the effect of oral processing behaviours and food texture on self-reported hunger, fullness and satiety outcomes.

\section{Conflict of Interest}

There is no conflict of interest 